\title{
Modern Rheology on a Stock Market: Fractional Dynamics of Indices
}

\author{
M. KozŁowska And R. KutneR \\ Division of Physics Education, Institute of Experimental Physics, Department of Physics, Warsaw University \\ Smyczkowa Str. 5/7, PL-02-678 Warsaw, Poland
}

(Received January 29, 2010)

\begin{abstract}
This paper presents an exactly solvable (by applying the fractional calculus) the rheological model of fractional dynamics of financial market conformed to the principle of no arbitrage present on financial market. The rheological model of fractional dynamics of financial market describes some singular, empirical, speculative daily peaks of stock market indices, which define crashes as a kind of phase transition. In the frame of the model the plastic market hypothesis and financial uncertainty principle were formulated, which proposed possible scenarios of some market crashes. The brief presentation of the model was made in our earlier work (and references therein). The rheological model of fractional dynamics of financial market is a deterministic model and it is complementary to already existing other ones; together with them it offers possibility for thorough and widespread technical analysis of crashes. The constitutive, fractional integral equation of the model is an analogy of the corresponding one, which defines the fractional Zener model of plastic material. The fractional Zener model is the canonical one for modern rheology, polymer physics and biophysics concerning non-Debye relaxation of viscoelastic biopolymers. The useful approximate solution of the constitutive equation of the rheological model of fractional dynamics of financial market consists of two parts: (i) the first one connected with long-term memory present in the system, which is proportional to the generalized exponential function defined by the Mittag-Leffler function and (ii) the second one describing oscillations (e.g. beats or oscillations having two slightly shifted frequences). The shape exponent leading the Mittag-Leffler function, defines here the order of the phase transition between bullish and bearish states of the financial market, in particular, for recent hossa and bessa on some small, middle and large stock markets. It happened that this solution also successfully estimated some long-term price dynamics on the hypothetical market in United States.
\end{abstract}

PACS numbers: 89.20.-a, 89.65.-s, 89.65.Gh, 89.90. $+\mathrm{n}$

\section{Introduction}

The bubbles and crashes are considered as unavoidable elements of stock market dynamics. They play a key role for capitalistic, competitive free markets $[1-4,5]$. Therefore bubbles and crashes are the natural subject of thorough and widespread studies of economists, sociologists, psychologists and recently, econo- and sociophysicists. The most fruitful seems to be the concept of the discrete scale invariance applied to stock markets and considering their crashes as a kind of criticality. As a consequence the dynamics of the market within the scaling region (i.e. in the region preceding a crash) can be described by scale-free laws containing logarithmic periodicities [6-16] (and references therein). The major achievement of the approach makes possible a forecasting of crash times.

There are many other fruitful analogies between the dynamics and/or stochastics of complex physical and economical or even social systems [17-25]. The methods and algorithms that have been explored for describing physical phenomena become an effective background and inspiration for very productive methods and algorithms used in analysis of economical, in particular the financial empirical data $[16,26]$.
Our concept is to consider only well developed temporal, non-exponential, speculative peaks of stock market indices. It relates in some sense to the idea of Eliezer and Kogan [9] (and references therein), which distinguishes the dynamics of the market in a crashing phase, from the one in the quiescent phase. In real market traders may form groups which then share information and act in coordination. This is the result of mutual interaction between traders leading to herding; groups may trade with each other through some centralized market procedure. We take into consideration the herding effect by way complementary to the approach already developed by percolation models $[5,27]$.

The solution supplied by our rheological model of the fractional dynamics of financial market (RMFDFM) is complementary to the power-law superposed with log-periodic oscillations $[2,16]$. We applied it here to describe some empirical data proceeding a crash, which cannot be successfully handled by the latter approach. Both approaches can constitute together a solid base for forecasting burdened with reduced risk.

The analysis was supported by the non-Debye or non-exponential relaxation processes observed in stress-strain relaxation of viscoelastic materials [28-33] as well 
as in the tick-by-tick empirical data for FUND future contract prices traded at LIFFE London [34].

In this paper we study, by way of examples, some mentioned above peaks and concern recent hossa and bessa:

(i) on emerging market: the daily closings of the historical Warsaw Stock Exchange (WSE) index WIG. We can assume that the dynamics of the WSE is typical for an emerging financial market of small size.

(ii) Besides, we study the Deutscher Aktien Index (DAX) as the index typical for middle stock markets and

(iii) Down Jones Industrial Average (DJIA) and SCI* indices as representative ones for large stock markets.

\section{Non-Debye relaxation}

It is important to know that the non-Debye relaxation may arise from memory, i.e. the underlying fundamental processes are of non-Markovian type. It was shown that fractional calculus is quite natural way of incorporating memory effects. The power-law kernel defining the fractional relaxation equation presents a long-term memory. The function that plays a dominating role in fractional relaxation problems is the Mittag-Leffler (ML) function [31]:

$$
\begin{aligned}
& E_{\alpha}\left(-\left(\frac{\left|t_{\mathrm{c}}-t\right|}{\tau}\right)^{\alpha}\right)=\sum_{n=0}^{\infty} \frac{\left[-\left(\left|t_{\mathrm{c}}-t\right| / \tau\right)^{\alpha}\right]^{n}}{\Gamma(1+\alpha n)}, \\
& \quad \alpha>0
\end{aligned}
$$

which is a straightforward generalisation of the exponential one (obtained for $\alpha=1$ ) and complementary to the famous Tsallis $q$-exponent [3] (here $t$ is time and $t_{\mathrm{c}}$ is the localization of the turning point from raising to falling parts of the ML function while $\alpha$ is the shape exponent). The ML function allows interpolation [1] between the corresponding stretched exponential function for short-time limit and power-law decay for asymptotic time (when $\alpha<1$ ); the former plays a crucial role in our analysis.

Before we go to financial markets, we pay our attention, for example, to the market of houses and parcels in U.S. As it is seen in Fig. 1, empirical data proceeding from this market is well fitted by the Mittag-Leffler function (red curve) even near to the turning point (i.e. to the point where hossa changes to bessa). The values of parameters of ML function obtained here are as follows: the localization of the turning point (maximum) is at $t_{\mathrm{c}}=231$ months, i.e. at March'07, which is placed exactly at the maximum of empirical data, the relaxation

\footnotetext{
* We chose Shanghai Composite Index intead of the Nikkei index as the latter too much oscillates.
}

time $\tau=95$ months, the shape parameter $\alpha=0.60$. Let us note that for $\alpha<1$ both derivatives (i.e. left- and right-sided) of the ML function diverges at $t_{\mathrm{c}}$ according to the power law, which means that return also accordingly diverges [1]. Hence, at $t_{\mathrm{c}}$ we deal with analogy of the first order phase transition. The corresponding predictions of exponential function (green curve) and stretched exponential one (blue curve) were also shown there for comparison $^{\dagger}$.

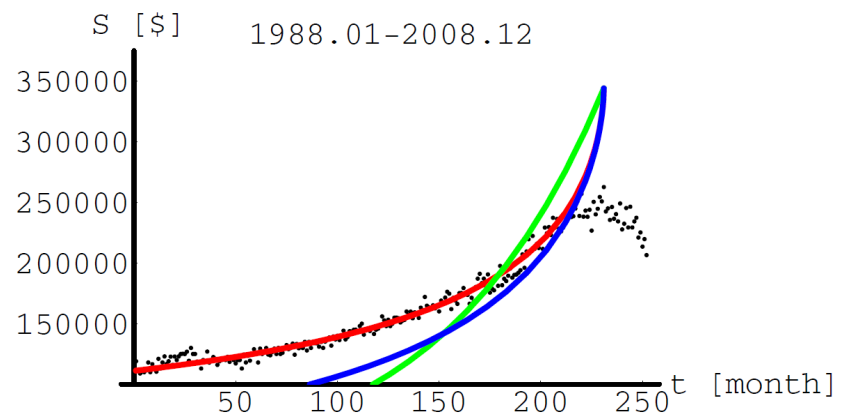

Fig. 1. The median, $S$, of prices of houses and parcels sold in U.S. between January 1988 and December 2008 (the unit of timescale is chosen here as one trading month). The empirical data (marked by dots) were downloaded from the internet address http://www.economagic.com/ (solid curves were defined in the main text).

Quite often the ML function appears both in the stochastic and deterministic modelling of disordered systems. The canonical example of the former may be the continuous-time random walk model (CTRW) used in the context of a financial market (Refs. [23-34, 35], while the latter - already mentioned above fractional relaxation equation describing relaxation of viscoelastic materials.

\section{Rheological model of fractional dynamics of financial market}

Presentation of our phenomenological, deterministic RMFDFM consists of two stages:

(i) Formulation of a linear ordinary differential equation (LODE) of the first order; this equation describes the index relaxation on an ideal market (considered in Sect. 3.1), i.e. only the exponential relaxation of an auxiliary index. By term "relaxation" we understand here and below in the text the relaxation both in forward and backward time-directions (of course, this backward relaxation of the index is equivalent to its increase in forward time-direction).

\footnotetext{
$\dagger$ For both exponential and stretched exponential functions relax-
} ation time is given here by $\tau[\Gamma(1+\alpha)]^{1 / \alpha}=79$ months. 
(ii) Fractional generalization of the LODE by using the replacing transformation that changes the above mentioned differential equation to a more general fractional form (including, by definition, the long-term memory). Thus we are able to model the non-exponential (non-Debye) relaxation of the empirical index on a real market within the range of speculative peaks. For example, the left path of the peak can initially increase very slowly and accelerates strongly toward the end of the peak, forming singularity of the first order ${ }^{\ddagger}$. The right paths can have an opposite dependence on time, i.e. initially rapidly decreases and finally very slowly. Of course, we also consider singularities of higher orders.

These stages define the general strategy, which was supplied by physical papers [28-30, 36-38] concerning rheological problems. The strategy was used for description of the non-Debye relaxation of viscoelastic materials and constitutes the basis for developing the fractional solid model (FSM). There are several versions of FSM [28] based on the so-called "fractional elements" defined by different mechanical arrangements of springs (i.e. elastic elements) and dashpots (i.e. friction ones), which are their fundamental structural quants. Such arrangement defines the rheological (macroscopic) properties of a solid, however several different arrangements can define the same properties.

The RMFDFM considers spring-dashpot pair as an analog of a single trader (investor), where spring symbolizes trader's activity and dashpot his aversion to risk $^{\S}$. If spring is stretched it means that investor buys stocks, if it is contracted then stockes are sold, otherwise (when spring leaves unchanged) the trader is waiting (or is doing nothing). The dashpot always acts (due to the friction or aversion to any risk) against any trader's activity. Hence, arrangement of spring-dashpot pairs defines a network of investors, which forms a social cooperative structure for a given stock market. The model is related to field called behavioural finance since it somehow incorporates the psychological motivation of investor's behaviour.

In our case the transition from stage (i) to stage (ii) means that the system under considerations changes from an ideal and unrealistic one to a realistic, complex system, where memory plays an essential role. This memory is defined by the integral, long-term kernel, analogously as it was done in terms of the FSM (cf. Eq. (3.13) in Sect. 3.3). By using our approach we described well developed speculative peaks concerning recent bessa and hossa present on small, middle and large financial markets.

\footnotetext{
$¥$ We say the function has singularity of $n$-th order at given point if its $n$-th order derivative diverges at this point but ones of all lower orders do not.

$\S$ This definition of trader does not exclude pathological possibilities that only the spring or only the dashpot represent some trader.
}

\subsection{Evolution of the auxiliary index}

The considerations given in this section (in particular, concerning Eq. (3.11)) realize at the present the stage (i) (defined at the beginning of Sect. 3), i.e. the ideal stock market dynamics (relaxation) for given path of the speculative peak.

\subsubsection{Basic assumptions of the model}

The RMFDFM is based on the following fundamental assumptions:

$\mathrm{A}_{1}$ ) In the range of well-defined speculative peaks (consisting of hossa and bessa paths), the dominating behaviour of the stock market results from traders' activities whose strategies are based only on a direct (on-line) observation of the market state. They are conducting a technical analysis of dynamics of stock market indices and the volume of the corresponding assets and undertake decision. They are called technical traders, chartists or noise traders. Of course, traders can mutually communicate without any delay (e.g. by using phones) and exchange informations.

The reason why technical traders dominate the stock market within the range of speculative peaks is caused, for example, by the time limitation as decision should be undertaken relatively quickly; there is no time for fundamental analysis of companies (quoted on a given stock market) conducting by fundamental traders (fundamentalists or rational traders), usually operating on much longer time horizon.

$\mathrm{A}_{2}$ ) For quantitative, technical analysis of the stock market we introduce two instantaneous, non-negative macroscopic (macroeconomic) variables $X(t)$ and $V(t)$ as basic, mutually independent quantities, where the first variable is the relative value of the stock market index while the second one is the relative volume of trade of those companies which constitutes this index; these time-dependent variables vanish only when they are equal to their background values (as usual, variable $t$ means the trading time). These basic variables can be directly, on-line monitored by chartists; this is the principal constraint which must subject any basic variable.

$\mathrm{A}_{3}$ ) By using basic variables we are able to express any other ones, for example, the instantaneous excess demand $U(t)$. This quantity, although recorded in the book of orders, does not make accessible for traders. We assume it in the form of a linear combination

$$
U(t)=a_{0} X(t)+b_{0} V(t),
$$

where, as usual, excess demand is a difference

$$
U(t) \stackrel{\text { def. }}{=} D(t)-S(t)
$$

between instantaneous demand $D(t)$ ( $\geq 0$ ) and supply $S(t)(\geq 0)$ (while $a_{0}$ and $b_{0}$ are time-independent coefficients). The relative volume of trade of stocks

$$
V(t) \stackrel{\text { def. }}{=} \min [D(t), S(t)] \text {. }
$$

Accordingly, quantities $D(t)$ and $S(t)$ denote the number of calls and puts transactions for stocks, respectively, at closing price. Let us note that the time step $\Delta t$ was assumed here as one trading day (i.e. $\Delta t=1 \mathrm{td}$ ) so $t$ 
counts trading days. This discretization of time justifies our assumption, given by Eq. (3.1), that both types of transactions (i.e. calls and puts ones) are always counted together (at the same time) with simultaneous observations of both basic variables.

To consider the relaxation of the auxiliary index we complete Eq. (3.1) with the differential equation, which shows an instantaneous, and therefore unrealistic (idealized), relation to the financial market. Namely,

$\mathrm{A}_{4}$ ) we can assume, in agreement with assumption $\mathrm{A}_{2}$, that the value of each basic variable in the nearest future is expressed by the linear combination of their current values; hence, we can write

$$
V(t+\Delta t)+e X(t+\Delta t)=c_{0} V(t)+d_{0} X(t),
$$

where $c_{0}$ and $d_{0}$ are time-independent coefficients.

It is straigtforward to derive from Eq. (3.4) the following differential equation:

$$
\frac{\mathrm{d} V(t)}{\mathrm{d} t}=C_{0} V(t)+D_{0} X(t)+E \frac{\mathrm{d} X(t)}{\mathrm{d} t},
$$

where coefficients $C_{0}=\frac{c_{0}-1}{\Delta t}, D_{0}=\frac{d_{0}-e}{\Delta t}$ are rates and $E=-e$ is a kind of relocation coefficient while " $\Delta$ " in Eq. (3.4) was replaced by its limited, infinitesimal operator "d".

By combining Eqs. (3.5) and (3.1) we eliminate the $V(t)$ variable with Eq. (3.5) and obtain the searched equation of ideal market dynamics in the form,

$$
U(t)+\tau_{0} \frac{\mathrm{d} U(t)}{\mathrm{d} t}=G_{0} \tau_{0} \frac{\mathrm{d} X(t)}{\mathrm{d} t}+G_{e} X(t),
$$

where coefficients

$$
\begin{aligned}
& \tau_{0} \stackrel{\text { def. }}{=}-\frac{1}{C_{0}}, \quad G_{0} \stackrel{\text { def. }}{=} a_{0}\left(1-B_{0} E\right), \quad B_{0}=-b_{0} / a_{0}, \\
& G_{e} \stackrel{\text { def. }}{=} a_{0}\left(1+B_{0} D_{0} / C_{0}\right),
\end{aligned}
$$

have additional, rheological interpretation (see below) since Eq. (3.6) is an analogy of the constitutive equation of the canonical rheological Zener model of plastic material [30]. This is the reason why we derived dynamics equation in such a form, by eliminating the directly measurable variable $V(t)$ instead of unmeasurable $U(t)$ one . As we will see, it makes possible the transition from an ordinary to the fractional differential equation, exactly in the same way as it was made in the modern rheology. Indeed, the latter equation is the fundamental one of modern rheology and in our case gives the possibility to consider peaks' non-exponential relaxation on a real market.

Let us note that Eq. (3.6) is a generalization of the canonical equation of the traditional economy, where change of price per unit time is proportional to the excess demand. However, the study of the former equation is not the aim of this work; our aim is its further generalization.

\footnotetext{
I Though the variable $V(t)$ is directly measurable, it strongly fluc-
} tuates which makes fit by any function very doubtful.

\subsection{Analogy to the standard linear solid model}

Equation (3.6) defines a model which can be considered as an analog of the standard linear solid model or Zener model of viscoelastic materials $[30,37,38]$. In this model the stress $(\sigma)$-strain $(\varepsilon)$ relationship is given originally by the following linear first order differential equation, the so-called rheological constitutive equation $(\mathrm{RCE})$

$$
\sigma(t)+\tau_{0} \frac{\mathrm{d} \sigma(t)}{\mathrm{d} t}=G_{0} \tau_{0} \frac{\mathrm{d} \varepsilon(t)}{\mathrm{d} t}+G_{e} \varepsilon(t),
$$

where $\tau_{0}$ is the transition time from elastic to plastic behaviour as only for $\tau_{0}$ sufficiently large, the deviation from Hooke law appears (then both derivatives can play the role), parameter $G_{e}$ is an elastic or low frequency modulus (the Young modulus) and $G_{0}$ is a plastic or high frequency modulus since only when it is nonvanishing the time-dependence of the strain influences the dynamics. Let us note that viscosity $\eta$ was defined within the standard linear solid model in [28, 29, 37, 38,] as follows $\eta \stackrel{\text { def. }}{=} \tau_{0}\left(G_{0}-G_{e}\right)$, which (in the financial context) can assume both negative and positive values (cf. also a short remark in Sect. 4).

By comparing Eqs. (3.6) and (3.8) we obtain correspondence between dynamic quantities of both models, which is shown in Table I. Hence, coefficients present in Eq. (3.6) have the meaning analogous to corresponding ones in Eq. (3.8), if they are non-negative.

TABLE I

Correspondence between an ideal stock market and the Zener solid model.

\begin{tabular}{c|c}
\hline Stock market & Zener solid model \\
\hline stock market index $X(t)$ & strain $\varepsilon(t)$ \\
excess demand $U(t)$ & stress $\sigma(t)$ \\
volume of trade $V(t)$ & temporal temperature $T(t)$
\end{tabular}

The convenient mechanical formulation of the Zener model $[28,36]$ consists of the Maxwell element connected in parallel with a spring ${ }^{* *}$ (it was assumed that springs always obey Hooke's law). Let us note that the Maxwell element consists of the spring and the dashpot (obeying Newton's law for viscous fluid) arranged in a sequential manner. This arrangement shows a simple spatial separation of the solid (elastic) and the fluid (viscous) aspects; it is, however, too specific to describe the most viscoelastic materials, e.g. such as biopolymers. Fortunately, the fractional solid model (sketched in Sect. 3.3), whose mechanical representation is given by a hierarchical arrangement of a number (in general infinite) of

\footnotetext{
** It should be added that springs and dashpots can represent not only psychological states of single traders but also groups of
} traders. 
springs and dashpots ${ }^{\dagger \dagger}$, is already sufficient [28, 36] to describe the non-Debye relaxation observed in the well known experiment, where stress relaxation under constant strain was measured. In our approach the spring represents a purely emotional or irrational investor's behaviour $^{\ddagger \ddagger}$ (an undamped activity) while the dashpot defines a purely rational one (fear or aversion to risk). Such an interpretation creates the possibility of construction of the mechanical model of an auxiliary and real stock markets.

\subsection{Conjecture: a transition to real market}

The considerations given in this and next sections (particularly Eq. (3.13)), realize the most important stage (ii) of our strategy defined at the beginning of Sect. 3. Before we go to the conjecture completing a transition from an ideal to real market, we give a motivation.

3.3.1. Influence of the past and future expectations on the current situation

Equation (3.6) describes only the present, temporal situation which is, however, the particular case; the more general one takes into account both the influence of the past events and future expectations on the current state of the market. First of all, to generalize Eq. (3.6), our initial Eqs. (3.1) and (3.4) should be extended. We can write them as follows:

$$
\begin{aligned}
& U(t)=a_{0} X(t)+b_{0} V(t) \\
& +\sum_{k=1}^{K(t)}\left[a_{k}^{-} X(t-k \Delta t)+b_{k}^{-} V(t-k \Delta t)\right] \\
& +\sum_{n=1}^{N(t)}\left[a_{n}^{+} X(t+n \Delta t)+b_{n}^{+} V(t+n \Delta t)\right],
\end{aligned}
$$

and

$$
\begin{gathered}
V(t+\Delta t)+e X(t+\Delta t)=c_{0} V(t)+d_{0} X(t) \\
+\sum_{k=1}^{K(t)}\left[c_{k}^{-} V(t-k \Delta t)+d_{k}^{-} X(t-k \Delta t)\right] \\
+\sum_{n=1}^{N(t)}\left[c_{n}^{+} V(t+n \Delta t)+d_{n}^{+} X(t+n \Delta t)\right],
\end{gathered}
$$

where coefficients denoted by the upper index "-" concern the past while these with "+" one concern the future. Of course, the generalization of differential Eq. (3.5), which we can directly derive from Eq. (3.10), also contains (in the cumulative form) terms responsible for past and future events. The same concerns the generalization

t† Note that different hierarchical arrangements of springs and dashpots were discovered which lead to the same fractional relaxation Eq. [28] (and refs. therein). In the context of the stock market this means that we have to deal with bifurcation of invested capital structure which depends on different strategies assumed by investors.

¥ In psychology the terminology "affected driven activity" or "automatic activity" is more frequently used. of Eq. (3.6), which we can easily obtain by combining Eqs. (3.9) and (3.10); however, this generalized equation would be too complicated to solve it. Therefore, to obtain a simplified equation which contains both past and future events we indeed utilize the approach developed in the frame of modern rheology.

\subsubsection{Conjecture: constitutive equation of fractional dy-} namics

Equation (3.6) is indeed the one which we generalize here into the fractional form according to the recipe elaborated within the modern rheology. This equation is integrated over time to yield

$$
\begin{aligned}
& X(t)-X(0)=-\frac{1}{\tau_{1}}{ }_{0} D_{t}^{-1} X(t)+\frac{1}{G_{0}} \frac{1}{\tau_{0}}{ }_{0} D_{t}^{-1} U(t) \\
& +\frac{1}{G_{0}}[U(t)-U(0)],
\end{aligned}
$$

where $\tau_{1} \stackrel{\text { def. }}{=} \tau_{0} G_{0} / G_{e}$ and definition of an inverse derivative of the first order was used; the definition of its general $n$-th order version (for $n=1,2,3, \ldots$ ) has a useful form given by the Cauchy formula of repeated integration

$$
\begin{gathered}
t_{0} D_{t}^{-n} f(t) \stackrel{d f .}{=} \int_{t_{0}}^{t} \mathrm{~d} t_{n-1} \int_{t_{0}}^{t_{n-1}} \mathrm{~d} t_{n-2} \ldots \\
\int_{t_{0}}^{t_{2}} f\left(t_{1}\right) \mathrm{d} t_{1} \int_{t_{0}}^{t_{1}} f\left(t_{0}^{\prime}\right) \mathrm{d} t_{0}^{\prime} \\
=\frac{1}{\Gamma(n)} \int_{t_{0}}^{t} \mathrm{~d} t^{\prime}\left(t-t^{\prime}\right)^{n-1} f\left(t^{\prime}\right) .
\end{gathered}
$$

There are several definitions of fractional differentiation and integration [33]. In what follows we are dealing strictly with the Riemann-Liouville (RL) fractional calculus. The RL fractional integration (integral operator), ${ }_{t_{0}} D_{t}^{-\alpha} f(t)$, of arbitrary order $\alpha(>0)$ of function $f(t)$ is a straightforward generalization of Eq. (3.12), where in the third row of Eq. (3.12) exponent $n$ was simply replaced by $\alpha[33]$.

The fractional generalization of Eq. (3.11) is performed, analogously as it was done for viscoelastic materials, by replacing expressions $\tau_{0}^{-1} t_{t_{0}} D_{t}^{-1} U(t)$ and $\tau_{1}^{-1}{ }_{t_{0}} D_{t}^{-1} X(t)$ by $\tau_{0}^{-\alpha}{ }_{t_{0}} D_{t}^{-\alpha} U(t)$ and $\tau_{1}^{-\alpha}{ }_{t_{0}} D_{t}^{-\alpha} X(t)$ ones, respectively, where the fractional exponent $\alpha$ is a free but most important shape parameter (exponent). Hence, we obtained a fractional integral equation, called further a constitutive equation of fractional dynamics (CEFD), which is able to describe both independent paths of speculative peaks

$$
\begin{aligned}
& X(y)-X(0)=-\left(\tau_{1}\right)^{-\alpha}{ }_{0} D_{y}^{-\alpha} X(y) \\
& +\frac{1}{G_{0}} \tau_{0}^{-\beta}{ }_{0} D_{y}^{-\beta} U(y) \\
& +\frac{1}{G_{0}}[U(y)-U(0)], \quad \alpha, \beta>0,
\end{aligned}
$$

where the independent variable 


$$
y=\left\{\begin{array}{rr}
t_{\mathrm{c}}-t, & \text { for } \text { retrospective relaxation } \\
& \left(\text { i.e. for hossa) }: t \leq t_{\mathrm{c}},\right. \\
t-t_{\mathrm{c}}, & \text { for }(\text { prospective) relaxation } \\
& \text { (i.e. for bessa) } t \geq t_{\mathrm{c}},
\end{array}\right.
$$

and $X(0)$, which is the maximal value of the index, is known from the initial condition. As both paths of any peak are assumed to be independent, we consider all parameters present in Eq. (3.13) as (in general) different for different paths.

For (prospective) relaxation the first term on the right hand side (rhs) of Eq. (3.13) is sensitive to the past due to the algebraic, integral kernel included there. The second term on the rhs of this equation desribes explicitly the summarized financial market influence on the index; the third term gives its instantaneous influence.

However, for retrospective relaxation situation is more complicated since Eq. (3.13) presents relaxation for inversed time, where current situation depends on future events again by an algebraic integral kernel. Of course, both types of relaxation are compared in this work with some empirical data.

Let us note that the pure fractional (prospective or retrospective) relaxation equation, i.e. the homogeneous one, is given by Eq. (3.13), where we put $1 / G_{0}=0$ or/and $U=0$ or by assuming $U$ proportional to $X$ for $\alpha=\beta$ and proper choices of three constant values.

\subsubsection{Comments on "mechanical" realization of consti-} tutive equation of fractional dynamics

Equation (3.13) (or CEFD) is an analogy of the corresponding fractional RCE describing fractional generalization of the Zener model of plastic material. In this generalized model the constitutive, mechanic elements (springs and dashpots) were replaced by fractional elements (FE) realized by particular spring-dashpot arrangements. These arrangements form particular ladders, trees and fractal networks (cf. corresponding figures in [28] and in references therein), which in the limit of infinite number of constitutive elements are physical realizations of the simplest fractional constitutive differential equation (Eq. (66) in [28]) being an interpolation between Hooke law and Newton one. Each FE is characterized by its own sequence of spring constants and viscosities.

Transition from Zener model to its fractional counterpart means that both springs and dashpot were replaced by fractional elements: two of them are connected in series (called fractional Maxwell element) and it is (as a whole element) parallel to the third FE. Thus, we have to deal with three groups of cooperative investors which is a reminiscence of an income distribution in society, where (roughly speaking) three essentially different prosperity groups were discovered [39-42]. Nevertheless, it is still a challenge to choose arrangements, which properly map microscopic cooperative structures of stock markets.

\subsection{Solution of the fractional initial value problem}

The explicit form of function $U$ required by Eq. (3.13) was suggested by, the commonly observed, oscillatory de- pendence of indexes on time. Hence, we solve the fractional initial value problem (3.13) by simply assuming that

$$
\begin{aligned}
& U(y)=\frac{U(0)}{2}[\exp (\mathrm{i}(\omega-\Delta \omega) y) \\
& \quad+\exp (\mathrm{i}(\omega+\Delta \omega) y)] .
\end{aligned}
$$

By applying the Laplace transform of the RL fractional integral operator (given by Eq. (A.5) in [32]) we easily obtain the Laplace transformation of Eq. (3.13). Next, by introducing the Laplace transform of (3.15) into our equation and by applying the inverse Laplace transformation into the time domain we finally obtain, for $\alpha=\beta>0$, the required real part of the exact solution (cf. expression (A.1) in our earlier work [1]).

To compare the prediction of our model with empirical data it is sufficient to use only the lowest order terms in the exact solution (A.1), i.e. it is suficient to use the following approximate expression:

$$
\begin{aligned}
& \Re X(y) \approx\left(X_{0}+X_{1}\right) E_{\alpha}\left(-\left(\frac{y}{\tau_{1}}\right)^{\alpha}\right) \\
& -X_{1} \cos (\omega y) \cos (\Delta \omega y),
\end{aligned}
$$

where terms proportional to $\omega$ as well as $\Delta \omega$ were neglected since for empirical data considered in this work the frequencies obey $\omega, \Delta \omega \ll 1$ (if additionally $\Delta \omega \ll \omega$ we have to deal with a beat) and all coefficients and parameters are real; the following notation was used:

$$
\begin{aligned}
& X_{0} \stackrel{\text { def. }}{=} X(0), \\
& X_{1} \stackrel{\text { def. }}{=}-\frac{1}{G_{0}}\left(\frac{\tau_{1}}{\tau_{0}}\right)^{\alpha} U(0) .
\end{aligned}
$$

The composed (time-independent) quantity given by the second expression in (3.17) is considered as a single parameter whose absolute value was found in the next section to be by few order of magnitude greater than unity. By comparison predictions of expression (3.16) with empirical data we can say more about the bubble and crash dynamics.

\section{Comparison with empirical data, discussion, conclusions and intentions}

As a simple test of validity of our rheological model of fractional dynamics of financial market, one can fit the formula (3.16), where we set $\Delta \omega=0$, to empirical data forming recent hossa of index WIG (cf. Fig. 2). It is seen that the fit (depicted by the red curve and considered as a trend) is satisfactory and the distribution of empirical points around the trend seems to have the statistical character. However, parameters $\tau_{1}$ and $X_{1}$ are burdened with huge dispersions.

This case (observed within our deterministic approach) seems to be a typical one for any hossa. It suggests the existence of a financial uncertainty principle (FUP) of quantities substantial to reach a profit by any investor ${ }^{\S}$.

\footnotetext{
$\S \S$ Uncertainty, together with risk and profit, were thoroughly and
} 
This principle can be formulated as follows: among quantities whose values have to be known to reach a profit during the hossa, at least one is unmeasurable, i.e. its value cannot be determined with sufficient precision. Otherwise, the profit could be obtained without any risk which widespreadly reviewed in book [43]. would be in contradiction to the market paradigm saying that market eliminates the arbitrage opportunity. Fortunately, due to anticorrelations existing between these quantities, the summarized dispersion can be sufficiently small to make the total fit satisfactory.

\section{TABLE II}

Fit parameters describing recent peaks of typical main indices of small, middle and large stock markets (upper elements L and R labeling parameters, designate left and right paths of a peak, respectively).

\begin{tabular}{c|c|c|c|c}
\hline \hline Parameter & WIG & DAX & DJIA & SCI \\
\hline$t_{\mathrm{c}}^{\mathrm{L}}$ & $892 \pm 73$ & $969 \pm 1$ & $627 \pm 3$ & $350 \pm 2$ \\
$t_{\mathrm{c}}^{\mathrm{R}}$ & $810 \pm 0$ & $940 \pm 0$ & $640 \pm 0$ & $282 \pm 0$ \\
$\tau_{1}^{\mathrm{L}}$ & $105 \pm 420$ & $426 \pm 391$ & $333 \pm 38$ & $196 \pm 15$ \\
$\tau_{1}^{\mathrm{R}}$ & $272 \pm 20$ & $426 \pm 72$ & $165 \pm 191$ & $138 \pm 8$ \\
$\alpha^{\mathrm{L}}$ & $0.57 \pm 0.23$ & $0.52 \pm 0.03$ & $1.29 \pm 0.02$ & $1.39 \pm 0.02$ \\
$\alpha^{\mathrm{R}}$ & $1.562 \pm 0.025$ & $1.12 \pm 0.03$ & $1.938 \pm 0.575$ & $1.30 \pm 0.13$ \\
$\omega^{\mathrm{L}}$ & $0.004 \pm 0.001$ & $0.004 \pm 0$ & $0.011 \pm 0.0$ & $0.075 \pm 0.001$ \\
$\omega^{\mathrm{R}}$ & $0.043 \pm 0.001$ & $0.009 \pm 0.0$ & $0.030 \pm 0.070$ & $0.051 \pm 0.001$ \\
$\Delta \omega^{\mathrm{L}}$ & $0.0 \pm 0.0$ & $0.0 \pm 0.0$ & $0.022 \pm 0.0$ & $0.0 \pm 0.0$ \\
$\Delta \omega^{\mathrm{R}}$ & $0.007 \pm 0.0$ & $0.025 \pm 0.0$ & $0.040 \pm 0.070$ & $0.0 \pm 0.0$
\end{tabular}

TABLE III

Calibrating fit parameters describing peaks of the same indices as shown in Table II.

\begin{tabular}{c|c|c|c|c}
\hline \hline Parameter [p] & WIG & DAX & DJIA & SCI \\
\hline$X_{0}^{\mathrm{L}}+X_{1}^{\mathrm{L}}$ & $60081 \pm 85273$ & $4698 \pm 82$ & $3486 \pm 40$ & $4810 \pm 75$ \\
$X_{0}^{\mathrm{R}}+X_{1}^{\mathrm{R}}$ & $41963 \pm 334$ & $5464 \pm 70$ & $4010 \pm 110$ & $3846 \pm 39$ \\
$X_{1}^{\mathrm{L}}$ & $-8659 \pm 2352$ & $-763 \pm 35$ & $-332 \pm 28$ & $-217 \pm 13$ \\
$X_{1}^{\mathrm{R}}$ & $-2528 \pm 269$ & $-847 \pm 36$ & $-866 \pm 81$ & $153 \pm 16$
\end{tabular}

Moreover, we found that in the case of index WIG the shape exponent $\alpha^{\mathrm{L}}$ is smaller than 1 (having a reasonable small dispersion, cf. the corresponding number shown in Table II at the cross of the second column and sixth row). Hence, the fitted (solid red) curve accelerates when it moves toward $t_{\mathrm{c}}$, where its derivative diverges. It suggests that we have to deal at this singular point with the analog of the first order phase transition on the Warsaw Stock Exchange. Thus we can identify at least a part of the crash phase region as the one placed in the vicinity of the singularity, where (to good approximation) both curves (red and blue shown in Fig. 2) coincide. Within this region small change of time causes large change of index, which can be one of the sources of a huge dispersion of some parameters. Below, we extended this discussion by considering recent daily peaks of indices listed in
Sect. 1. Full peaks of indices WIG, DAX, DJIA and SCI were presented in subsequent Figs. 3-6 while corresponding fit parameters were shown in Tables II-IV (so far, in Table $\mathrm{V}$ important dates were presented concerning $t_{\mathrm{c}}$ 's shown in Table II). Again, satisfactory agreement with empirical data are observed, which even makes possible to draw some general conclusions.

It is seen from Table II that:

- the range of the hossa shape exponent $\alpha$ is larger than 0 and smaller than 2 for considered peaks. Moreover, for large stock markets (such as New York Stock Exchange (NYSE) and Shanghai ones) they are even larger than 1. For bessa shape exponents they are all larger than 1 . All these observations seem to be true also for other recent peaks of 


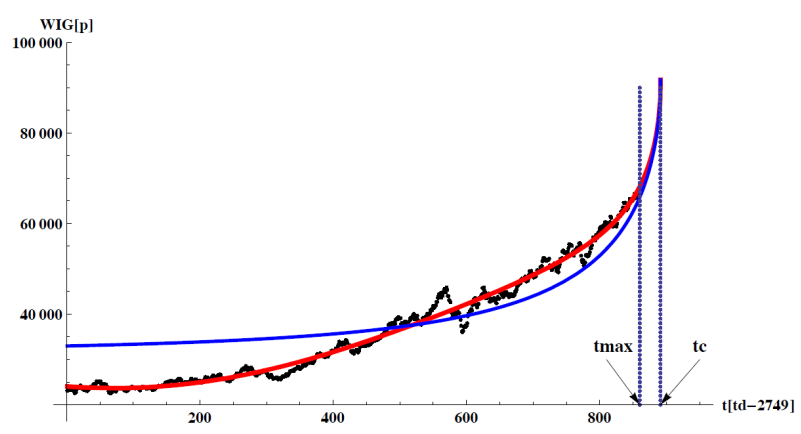

Fig. 2. Recent hossa of index WIG (the daily closing value measured in conventional points) dated from 2004.02.06 (or 2749 stock market session) to 2007.07.06 and consisting of 860 sessions. The empirical data are marked by black dots, the prediction of formula (3.16) for $\Delta \omega=0$ is given by red solid curve while blue solid curve is prediction of the stretched exponential function plotted for the same parameters (shown in Tables II and III) except parameter $X_{1}$ that was set to zero. The time $t_{\max }$ denotes the position of the empirical hossa's maximum and $t_{\mathrm{c}}$ - the location of the theoretical turning point (from hossa to bessa) common for both functions.

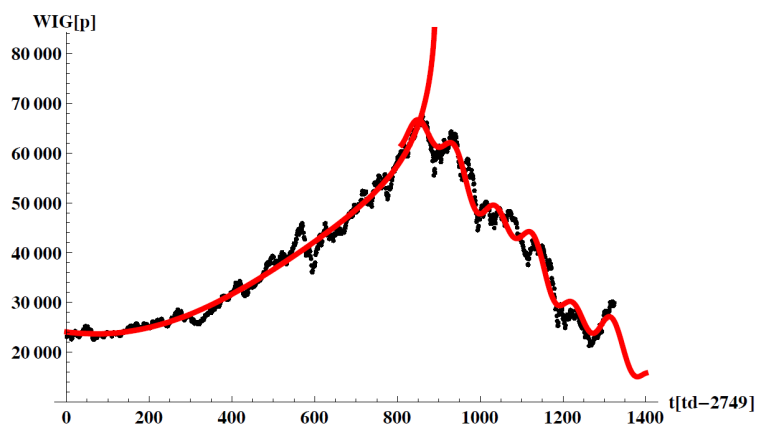

Fig. 3. Recent (full) peak of WIG extending from 2004-02-06 (or from the 2750th stock market session) to 2009-05-18 (or to the 4073rd session). A little overlap between the (theoretical) rising and falling paths at the top of the peak is seen. This is caused by the uncertainty concerning the theoretical beginning of the bessa and the assumption that both paths can be considered as independent ones. Here, the theoretical beginning of the bessa was assumed as 2007-04-24 (or the 3559th stock market session).

TABLE IV

Accuracy of the fit, where fit parameters were shown in Tables II and III.

\begin{tabular}{c|c|c|c|c}
\hline \hline Fit accuracy & WIG & DAX & DJIA & SCI \\
\hline$R_{\mathrm{L}}^{2}$ & 0.9986 & 0.9985 & 0.9996 & 0.9983 \\
$R_{\mathrm{R}}^{2}$ & 0.9985 & 0.9977 & 0.9971 & 0.9967
\end{tabular}

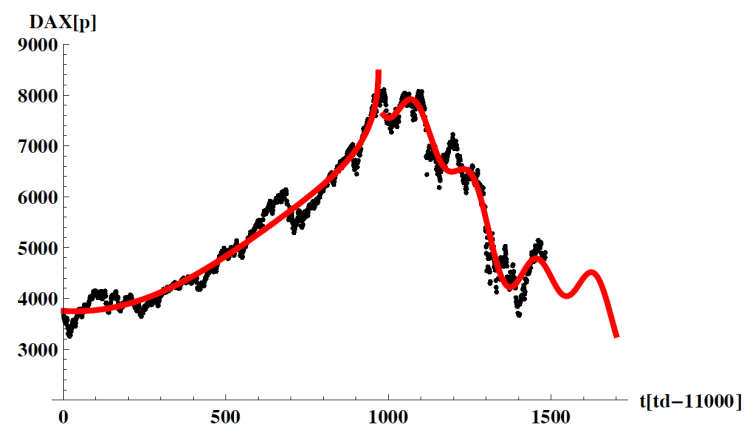

Fig. 4. Recent (full) peak of DAX extending from 2003-09-04 (or from the 11001st stock market session) to 2009-07-01 (or to the 12482nd session). The (theoretical) left path of the peak ends at 2007-07-13 (or ends at the 11985th session) while the right one begins from 2007-07-12 (or begins from the 11984th session). A little discontinuity of paths and their slight displacement is seen at the top of the peak. Again, this results from the uncertainty concerning the theoretical beginning of the bessa and treating of both paths as independent ones.

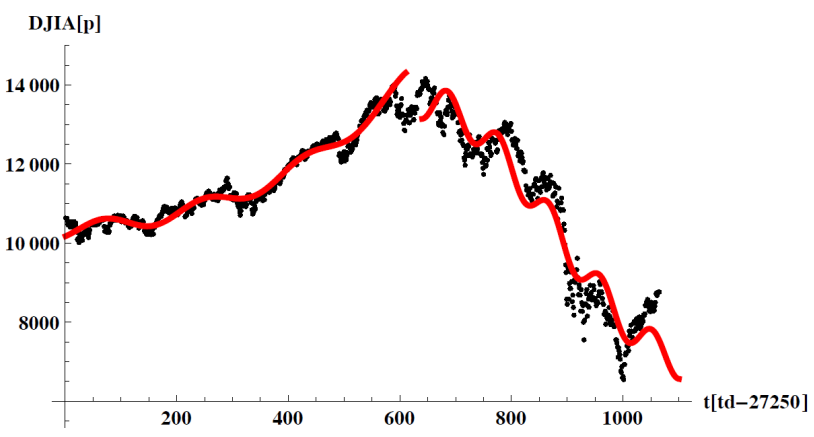

Fig. 5. Recent (full) peak of DJIA extending from 2005-03-16 (or from the 27251st stock market session) to 2009-06-09 (or to the 28315th session). The (theoretical) left path of the peak ends at 2007-09-12 (or ends at the 27877th stock market session) while the right one begins from 2007-10-01 (or begins from the 27890th session). A little discontinuity and displacement of theoretical (solid) curves, seen at the top of the peak, results from the fact that its both paths are considered as independent ones.

main stock market indices;

- localization of maxima, $t_{c}$, of hossa and bessa were found with relatively small dispersion in contradiction to fractional relaxation time ones, $\tau_{1}$. For most of peaks $t_{\mathrm{c}}^{\mathrm{R}}<t_{\mathrm{c}}^{\mathrm{L}}$ and for other ones $t_{\mathrm{c}}^{\mathrm{R}} \geq t_{\mathrm{c}}^{\mathrm{L}}$;

- all frequencies, $\omega$, and frequency shifts, $\Delta \omega$, are much smaller than 1 , which obeys a constraint required for application of the approximate solution (3.16).

It is evident from Table III that:

- the calibration (combined) parameter $X_{1}$ can assume both the positive and negative values, which 


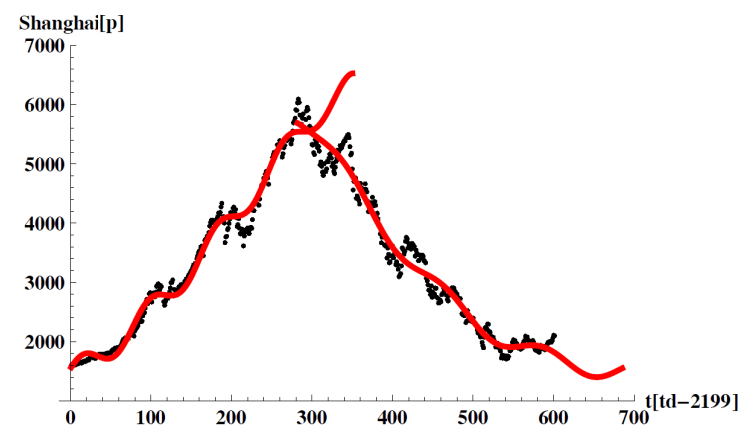

Fig. 6. Full peak of SCI extending from 2006-08-15 (or from the 2200 stock market session) to 2009-02-05 (or to the 2800 session), which much less oscillates in this range than the Nikkei. The (theoretical) left path of the peak ends at 2007-10-18 (or ends at the 2549th stock market session) while the right one begins from 2007-10-16 (or begins from the 2481st session). The overlap of theoretical (solid) curves observed at the top of the peak results, as usual, from uncertainty concerning the location of the hossa-bessa turning point and independence of both paths.

TABLE V

Dates which correspond to $t_{\mathrm{c}}^{\mathrm{L}}$ and $t_{\mathrm{c}}^{\mathrm{R}}$ shown in Table II.

\begin{tabular}{c|c|c|c|c}
\hline \hline Date & WIG & DAX & DJIA & SCI \\
\hline$t_{\mathrm{c}}^{\mathrm{L}}$ & $2007-07-06$ & $2007-07-13$ & $2007-09-12$ & $2008-01-22$ \\
$t_{\mathrm{c}}^{\mathrm{R}}$ & $2007-04-24$ & $2007-07-12$ & $2007-10-01$ & $2007-10-16$
\end{tabular}

depends on sign of the initial value $U(0)$ (cf. the second expression in (3.17)). Unfortunately, although we obtained $\alpha$ 's and $\tau_{1}$ 's directly from fits, it is impossible to separately derive parameters needed for calculation viscosities $\eta$ 's (i.e., parameters $\tau_{0}$ 's and $G_{e}$ 's, see remark in Sect. 3.3.2. concerning Eq. (3.11)). We can only say that we cannot exclude negative values of viscosities (which could occur if $\tau_{1}$ would be smaller than $\tau_{0} \boldsymbol{\Phi}$ ) that are related to positive feedbacks [2].

$>$ From Table IV it follows that:

- accuracies, $R^{2}$, of all fits are satisfactory as they are restricted to rather narrow range $0.9967 \leq R^{2} \leq$ 0.9996 .

We can conclude that:

- the rough short-term forecasting made by simple extrapolation of theoretical curves is, of course, possible (see, for instance, Fig. 4).

ฯ This conclusion results from the formula $\eta=G_{e}\left(\tau_{1}-\tau_{0}\right)$, which can be easily obtained from definitions of $\eta$ and $\tau_{1}$.

\subsection{Supplementary correspondence between the stock market and equilibrium thermodynamics}

The additional, useful correspondence between some stock market quantities and thermodynamic ones is given in Table VI, where $L_{0}$ is a free linear size when no stress is applied, $\Delta L$ is the change of the linear size under the applied stress, $T$ is (as usual) the temperature while $T_{\mathrm{C}}$ transition temperature and $p$ is a pressure. We did not consider here the thermodynamics of a fractional solid (which is interesting of itself), but only pay attention to some supplementary analogy between the stock market and thermodynamics; decisive here is the correspondence between the interest rate per unit time and the linear coefficient of expansion whose divergence gives indeed the criterion for the dynamic phase transition possible at the stock market.

\subsection{Unified equation for fractional relaxation, \\ log-periodic oscillations and Tsallis q-exponential function}

Since log-periodic oscillations and Tsallis $q$-exponential function were so successfully applied to description of the dynamics of stock market indices or returns the question arises whether is it possible to find a unified equation, which is able to cover all these three dynamics together***. In this subsection we perform a single step toward the answer for this question by extending the corresponding derivation given in [3].

In the course of extension we propose the generalized, unified fractional relaxation equation in the form

$$
\begin{aligned}
& \frac{\mathrm{d}^{\eta}\left(y^{\xi} X(y)\right)}{\mathrm{d}\left(y^{\alpha}\right)^{\eta}}=-F(y)[X(y)]^{q}, \quad y \geq 0, \\
& F(y) \stackrel{\text { def. }}{=}-\left(\gamma+\mathrm{i} \omega+\xi y^{\xi-1}\right) B,
\end{aligned}
$$

where all real quantities $\eta(>0), \xi(>0), \alpha(>0)$, $q, \gamma, \omega, B$ are independent of variable $y$ and the Caputo fractional derivative is defined by the Riemann-Liouville fractional calculus as follows:

$$
\frac{\mathrm{d}^{\eta} X(y)}{\mathrm{d} y^{\eta}} \stackrel{\text { def. }}{=}{ }_{0} D_{y}^{-(1-\eta)} X^{(1)}(y),
$$

where $X^{(1)}$ means the first derivative of $X$. The type of the solution $X_{\eta, \xi, \alpha, q}(y ; \gamma, \omega, B)$ of Eq. (4.1) decisively depends on values of exponents $\eta, \xi, \alpha, q$.

It is illustrative to present two essentially different groups of solutions; the first one, discussed already by Tsallis [3]

$$
\begin{aligned}
& X_{\eta, \xi=0, \alpha, q}(x ; \gamma=-1, \omega=0, B) \\
& \sim\left\{\begin{array}{cc}
E_{\eta}\left(-(y / \tau)^{\eta}\right), & x=y^{\eta}, \alpha=1, q=1, B=\tau^{-\eta}, \\
\exp \left(-(y / \tau)^{\alpha}\right), & x=y, \eta=1, q=1, B=\tau^{-\alpha}, \\
\exp _{q}(-\beta y), & x=y, \eta=1, \alpha=1, B=\beta,
\end{array}\right.
\end{aligned}
$$

and the second group concernig the $\log$-periodic oscillations:

\footnotetext{
*** This subsection was prepared under direct inspirations of Con-
} stantino Tsallis and Didier Sornette. 


$$
\begin{aligned}
& X_{\eta=1, \xi=1, \alpha=1, q=1}(x ; \gamma, \omega, B=1) \sim 1-\left[\left(1-\delta_{\gamma, 1}\right) B_{1} y^{\gamma}\right. \\
& \left.\quad+\delta_{\gamma, 1} B_{2} \ln (y)\right][1+C \cos (\omega \ln (y)+\phi)] .
\end{aligned}
$$

Of course, to find general solution of Eq. (4.1), which seems to be a challenge, some initial or/and boundary conditions are required.

Finally, we suppose the rheological model of fractional dynamics of financial market, together with above mentioned fits by $\log$-periodic oscillations and $q$-exponential function, can rationally decrease the risk of investment on a stock market since it allows to warn the investors before the stock market reaches a crash region. We hope that our approach will be an inspiration for futher applications, generalizations and study of not only the dynamics but also microscopic cooperative structures of stock markets.

TABLE VI

Correspondence between some stock market and thermodynamic quantities.

\begin{tabular}{c|c}
\hline \hline Stock market & Thermodynamics \\
\hline stock market index $X$ & linear size $L\left(=L_{0}+\Delta L\right)$ \\
$y=t-t_{\mathrm{c}}$ & $y=T-T_{\mathrm{C}}$ \\
returns per unit time $\frac{\mathrm{d} \ln X}{\mathrm{~d} y}$ & linear coefficient of expansion $\left(\frac{\partial \ln L}{\partial T}\right)_{p}$
\end{tabular}

returns per unit time $\frac{\mathrm{d} \ln X}{\mathrm{~d} y} \quad$ linear coefficient of expansion $\left(\frac{\partial \ln L}{\partial T}\right)_{p}$

\section{Acknowledgments}

The authors are very grateful to Didier Sornette and Constantino Tsallis for their valuable comments and suggestions. This work was partially supported by Polish Grant No. 119 obtained within the First Competition of the Committee of Scientific Research organized by National Bank of Poland.

\section{Appendix \\ Exact solution of the fractional initial value problem}

The real part of the exact solution of our fractional initial value problem (3.13) takes, for $\alpha=\beta$, the following form:

$$
\begin{aligned}
& \Re X(y)=\left(X_{0}+X_{1}\right) E_{\alpha}\left(-\left(\frac{y}{\tau_{1}}\right)^{\alpha}\right) \\
& -X_{1} \cos (\omega y) \cos (\Delta \omega y) \\
& -\omega X_{1}\left(1-\left(\frac{\tau_{0}}{\tau_{1}}\right)^{\alpha}\right) \int_{0}^{y} \sin \left(\omega\left(y-y^{\prime}\right)\right) \\
& \quad \times \cos \left(\Delta \omega\left(y-y^{\prime}\right)\right) E_{\alpha}\left(-\left(\frac{y^{\prime}}{\tau_{1}}\right)^{\alpha}\right) \mathrm{d} y^{\prime} \\
& -\Delta \omega X_{1}\left(1-\left(\frac{\tau_{0}}{\tau_{1}}\right)^{\alpha}\right) \int_{0}^{y} \cos \left(\omega\left(y-y^{\prime}\right)\right) \\
& \quad \times \sin \left(\Delta \omega\left(y-y^{\prime}\right)\right) E_{\alpha}\left(-\left(\frac{y^{\prime}}{\tau_{1}}\right)^{\alpha}\right) \mathrm{d} y^{\prime} .
\end{aligned}
$$

In all our calculations we used approximate solution given by the first and second rows in (A.1), i.e. we assumed that both $\omega$ and $\Delta \omega$ are at most of the order of 0.1 (cf. Table II) and the fraction $\left(\frac{\tau_{0}}{\tau_{1}}\right)^{\alpha}$ at most of the order of 1 , which makes our fit consistent (in spite of that, we have empirical data insufficient to verify the value of this fraction).

\section{References}

[1] M. Kozłowska, A. Kasprzak, R. Kutner, Int. J. Mod. Phys. C 19, 453 (2008).

[2] D. Sornette, Why Stock Markets Crash. Critical Events in Complex Financial Systems, Princeton University Press, Princeton 2003.

[3] C. Tsallis, Braz. J. Phys. 39, 337 (2009).

[4] B.M. Roehner, Patterns of Speculation. A Study in Observational Econophysics, Cambridge University Press, Cambridge UK 2002.

[5] M. Ausloos, in: Econophysics and Sociophysics. Trends and Perspectives, Eds. B.K. Chakrabarti, A. Chakraborti, A. Chatterjee, Wiley-VCH Verlag, Weinheim 2006, Ch. 9, p. 249.

[6] W.-X. Zhou, D. Sornette, Physica A 330, 543 (2003).

[7] W.-X. Zhou, D. Sornette, Physica A 330, 584 (2003).

[8] P. Gnaciński, D. Makowiec, Physica A 344, 322 (2004).

[9] S. Drożdż, F. Grummer, F. Ruf, J. Speth, Physica A 324, 174 (2003).

[10] S. Drożdż, J. Kwapień, P. Oświęcimka, J. Speth, Acta Phys. Pol. A 114, 539 (2008).

[11] D. Sornette, A. Johansen, J.-P. Bouchaud, J. Phys. I France 6, 167 (1996).

[12] D. Sornette, A. Johansen, Physica A 245, 411 (1997).

[13] D. Sornette, A. Johansen, Quant. Finance 1, 452 (2001).

[14] A. Johansen, D. Sornette, J. Risk 4, 69 (2001). 
[15] A. Johansen, Comment on Are financial crashes predictble? by L. Laloux, M. Potters, R. Cont, J.-P. Aguilar, J.-P. Bouchaud, Europhysics Lett. 60 (5), 809 (2002).

[16] D. Grech, Z. Mazur, Acta Phys. Pol. B 36, 2403 (2005).

[17] R. Badii, A. Politi, Complexity. Hierarchical Structures and Scaling in Physics, Cambridge University Press, Cambridge UK 1997.

[18] W. Paul, J. Baschnagel, Stochastic Processes. From Physics to Finance, Springer-Verlag, Berlin 1999.

[19] R.N. Mantegna, H.E. Stanley, An Introduction to Econophysics: Correlations and Complexity in Finance, Cambridge University Press, Cambridge UK 2000.

[20] J.-P. Bouchaud, M. Potters, Theory of Financial Risks. From Statistical Physics to Risk Management, Cambridge University Press, Cambridge UK 2001.

[21] K. Ilinski, Physics of Finance. Gauge Modelling in Non-Equilibrium Pricing, Wiley, Chichester 2001.

[22] E. Scales, R. Gorenflo, F. Mainardi, Phys. Rev. E 69, 011107 (2004).

[23] L. Sabatellil, S. Keating, J. Dudley, P. Richmond, Eur. Phys. J. B 27, 273 (2002).

[24] Special Issue \& Directory, Nonextensive Statistical Mechanics: New Trends, New Perspectives, Europhysicsnews 36/6 (2005).

[25] F. Schweitzer, Brownian Agents and Active Particles, Springer-Verlag, Berlin 2003.

[26] A. Bunde, J. Kantelhardt, Phys. Blätter 57, 49 (2001).

[27] R. Cont, J.-P. Bouchaud, Macroecon. Dyn. 4, 170 (2000).

[28] H. Schiessel, Chr. Friedrich, Blumen, in Applications of Fractional Calculus in Physics, Ed. R. Hilfer, World Scientific, Singapore 2000, Chap. 7, pp. 331376 .

[29] Th.F. Nonnenmacher, R. Metzler, Applications of Fractional Calculus in Physics, Ed. R. Hilfer, World Sci., Singapore 2000, Ch. 8, p. 377.
[30] N. Tschoegel, The Phenomenological Theory of Linear Viscoelastic Behavior, Springer-Verlag, Berlin 1989.

[31] R. Richert, A. Blumen, Disorder Effects on Relaxational Processes: Glasses, Polymers, Proteins, Eds. R. Richert, A. Blumen, Springer, Berlin 1994, Ch. 1, p. 1

[32] R. Metzler, J. Klafter, Phys. Rep. 339, 1 (2000).

[33] P.L. Butzer, U. Westphal, Applications of Fractional Calculus in Physics, Ed. R. Hilfer, World Sci., Singapore 2000, Ch. 1 p. 1.

[34] F. Mainardi, M. Raberto, R. Gorenflo, E. Scales, Physica A 287, 468 (2000).

[35] R. Kutner, F. Świtała, Qunatitative Finance 3, 201 (2003)

[36] H. Schiessel, A. Blumen, J. Phys. A: Math. Gen. 26, 5057 (1995)

[37] W.G. Glöckle, Th.F. Nonnenmacher, Macromolecules 246426 (1991).

[38] W.G. Glöckle, Th.F. Nonnenmacher, Rheol. Acta 33, 337 (1994)

[39] P. Richmond, S. Hutzler, R. Coelho, P. Reptowicz, in Ref. [9], Ch. 5, p. 131

[40] S. Iyazima, K. Yamamoto, in: Practical Fruits of Econophysics. Proceedings of the Third Nikkei Econophysics Symposium, Ed. H. Takayasu, Springer-Verlag, Berlin 2006, p. 344.

[41] K. Yamamoto, S. Miyazima, H. Yamamoto, T. Ohtsuki, A. Fujihara, in Ref. [40], p. 349.

[42] M. Jagielski, R. Kutner, Acta Phys. Pol. A 118, 615 (2010)

[43] E. Borgonovo, L. Peccati, in: Uncertainty and Risk, Eds. M. Abdellaoni, R.D. Luce, M.J. Machina, B. Munier, Springer-Verlag, Berlin 2007, Part I, p. 41. 